To appear in Proc. 2012 ICRA

\title{
Putting the Fish in the Fish Tank: Immersive VR for Animal Behavior Experiments
}

\author{
Sachit Butail, Amanda Chicoli and Derek A. Paley
}

\begin{abstract}
We describe a virtual-reality framework for investigating startle-response behavior in fish. Using real-time threedimensional tracking, we generate looming stimuli at a specific location on a computer screen, such that the shape and size of the looming stimuli change according to the fish's perspective and location in the tank. We demonstrate the effectiveness of the setup through experiments on Giant danio and compute the success rate in eliciting a response. We also estimate visual startle sensitivity by presenting the stimulus from different directions around the fish head. The aim of this work is to provide the basis for quantifying escape behavior in fish schools.
\end{abstract}

\section{INTRODUCTION}

The collective response of a fish school to a predator is a rapidly propagating wave of visual and hydro-acoustic information transmitted via local interactions that can travel faster than the speed of an approaching predator [1]. Escape behaviors such as these are part of group communication strategies that inspire the design of reactive multi-agent robotic systems [2], control algorithms [3], [4], and the design of autonomous systems [5].

Past studies characterizing animal behavior in response to visual cues involve presenting a visual stimulus to a subject animal that is not completely free to move in three dimensions [6], [7]. In [8], the constraint on position is relaxed by putting the animal, a crab, on a floating styrofoam ball. Similar technique may not be practical to implement with animals in groups.

Virtual reality provides a novel opportunity for high-output biological data collection and allows for the manipulation of sensory feedback. Virtual reality paradigms have been harnessed as an experimental tool to study spatial navigation and memory in rats [9], flight control in flies [10] and balance studies in humans [11]. A virtual-reality paradigm may be used to understand local interactions and quantify sensory volumes that ultimately lead to coordinated group behavior, inspiring the design of control laws for autonomous vehicles with limited sensing.

In this paper, we describe a virtual-reality setup for investigating startle behavior that relaxes the constraint on the animal motion by dynamically changing the perceived

S. Butail is a graduate student in the Department of Aerospace Engineering, University of Maryland, College Park, MD, 20742, USA sbutaileumd. edu

A. Chicoli is a graduate student in the Neuroscience and Cognitive Science Program, University of Maryland, College Park, MD, 20742, USA achicolieumd.edu

D. A. Paley is an assistant professor in the Department of Aerospace Engineering, University of Maryland, College Park, MD, 20742, USA dpaleyeumd. edu environment in response to the animal's position, orientation and velocity. The attractiveness of this automation is that it enables realistic behavioral experiments while allowing control over the animal's perceived environment. For example, by way of controlling where, how, and when to present a stimulus, this technique promises to investigate aspects of animal behavior such as visual perceptual volume, what qualifies as threat, and quorum sensing [12]. The basic framework is a visual feedback loop that automates the process of presenting the stimulus via a computer screen based on criteria on the animal's pose.

Fish-tank virtual-reality (VR) uses the viewer's head pose to dynamically update the display on a screen [13]. This concept is used in augmented reality systems with headmounted gear or visual tracking [13], [14]. A virtual-reality system changes the image on the screen based on the viewer's perspective. The importance of VR is unknown for animal behavior experiments. Preliminary results show an increased probability of response with virtual-reality stimuli as compared to non virtual-reality stimuli.

The contributions of this paper are (1) the development of an automated virtual-reality system for animal behavior experiments; (2) the demonstration of the effectiveness of a VR setup in eliciting a startle response in giant danio (Danio aequipinnatus), a common species of freshwater fish; and (3) the construction of a three-dimensional startle sensitivity map for a single danio based on its response to looming stimuli in a free-swimming environment.

Section II gives a background on the paper's biological significance, virtual reality and three-dimensional tracking. Section III describes the looming stimulus from a viewer's perspective and reviews the tracking algorithm for estimating three-dimensional position and orientation (pose), and velocity in real time. Section IV presents the experimental validation, materials and methods, and results. Section V summarizes the paper and discusses ongoing work.

\section{BACKGROUND}

This section discusses the biological significance of a virtual-reality framework in animal behavior experiments. It also describes the virtual-reality setup and three-dimensional object tracking.

\section{A. Biological Significance}

The startle response of fish to a perceived threat is used in the investigation of sensory-motor integration [15] and collective decision-making [16] and plays an important role in predator evasion and the collective dynamics of a fish 
school. Visual cues such as an approaching predator can elicit a startle response whether the cue is observed or not. The visual fields of fish play an important role in predator-prey interactions and, presumably, shoaling behavior. Despite the importance, visual fields of fishes have only been investigated in several fish species (see [17] for a review) and have not been characterized for a group of animals, including fishes. This paper expands on that work by behaviorally characterizing the receptive fields of Giant danios through the use of virtual reality.

The use of a virtual-reality design with real-time sensory feedback also enables the manipulation of a stimulus or scene during a trial without experimenter intervention, and provide a natural stimulus display. The use of natural stimuli is common for understanding a variety of sensory systems [18], [19]. These studies and many more have helped demonstrate the dynamic properties of sensory neuronal systems and have led to hypotheses on how these systems may be optimized.

\section{B. Virtual-reality setup}

The virtual-reality setup we use in our experiments comprises a single looming stimulus that approaches the viewer from a distance. The stimulus in our experiments is an onscreen representation of a three-dimensional moving object. The threat presented by a looming stimulus depends on its size, speed, and direction of motion. The perspective of the viewer determines how the object is presented on a computer screen. To represent the view on the computer screen we assume a perspective-projection model, i.e., an object appears larger as it gets closer. A limitation of the virtual-reality setup described here is that the scene is set for only one subject at a time. This limitation is in part due to the large perceptual range of fish visual field [20] as well as size of the screen.

Fig. 1 shows a schematic of the test environment. Let $\mathcal{I}$ be the inertial reference frame, $\mathcal{V}$ be the viewer frame and $\mathcal{S}$ be a frame fixed to the stimulus. The position $t \in$ $\mathbb{R}^{3}$ and orientation $R \in \mathbf{S O}(3)$ of a reference frame is represented in another frame by a $4 \times 4$ transformation matrix $T=\left[\begin{array}{cc}R & \boldsymbol{t} \\ \mathbf{0}^{T} & 1\end{array}\right]$. Let ${ }^{\mathcal{I}_{T}} T_{\mathcal{V}}$ represent the configuration of the viewer frame in the inertial frame and ${ }^{\mathcal{I}_{T}} T_{\mathcal{S}}$ represent the configuration of the stimulus frame in the inertial frame. The stimulus in the viewer frame is ${ }^{\mathcal{V}_{T}}={ }^{\mathcal{V}_{T_{\mathcal{I}}}}{ }^{\mathcal{I}} T_{\mathcal{S}}$, where ${ }^{\mathcal{V}_{T}} T_{\mathcal{I}}\left({ }^{\mathcal{I}} T_{\mathcal{V}}\right)^{-1}$.

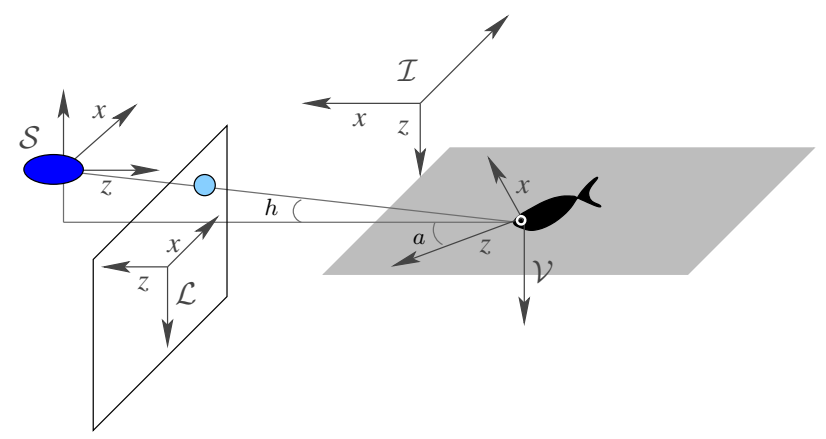

Fig. 1. Reference frames $\mathcal{V}$ (viewer), $\mathcal{S}$ (stimulus), $\mathcal{L}$ (screen) and $\mathcal{I}$ (inertial). The azimuth $(a)$ and elevation $(h)$ of the stimulus with respect to the fish are also shown.

\section{Three-dimensional tracking}

Tracking the three-dimensional position and orientation of the fish is required to present the stimulus to the fish. Tracking objects in three dimensions using cameras is a wellresearched problem [21], [22]. Each application motivates a different camera arrangement [23], [24], [25], but the basic approach stays the same. A single-camera system is attractive since the data can be processed in real-time without the need to synchronize between multiple views. Since a single camera may be unable to observe the three-dimensional position of an object, techniques that solve this problem include model-based tracking [21] and obtaining another perspective from a mirror [23] or shadows [26]. Real-time tracking capabilities depend on the frame rate, processing power, and the amount of data to be processed in each frame.

In a target-tracking framework, the state of a target at time $k$ is described by the vector $\boldsymbol{X}_{k} \in \mathbb{R}^{n}$. A measurement at time $k$ is denoted by $\boldsymbol{Z}_{k} \in \mathbb{R}^{m}$. The state $\boldsymbol{X}_{k+1}$ and measurements $\boldsymbol{Z}_{k+1}$ are related to the state $\boldsymbol{X}_{k}$ according to

$$
\begin{aligned}
\boldsymbol{X}_{k+1} & =\boldsymbol{F}\left(\boldsymbol{X}_{k}, \boldsymbol{w}_{k+1}\right) \\
\boldsymbol{Z}_{k+1} & =\boldsymbol{H}\left(\boldsymbol{X}_{k+1}, \boldsymbol{n}_{k+1}\right),
\end{aligned}
$$

where $\boldsymbol{F}$ represents the motion model, $\boldsymbol{H}$ represents the measurement model, and $\boldsymbol{w}$ and $\boldsymbol{n}$ are the instantaneous disturbance- and measurement-noise values. Given the state estimate $\hat{\boldsymbol{X}}_{k}$, estimation error $\hat{\boldsymbol{X}}_{k}-\boldsymbol{X}_{k}$ occurs due to noise and approximation in $\boldsymbol{F}$ and $\boldsymbol{H}$. The conditional probability of state $\boldsymbol{X}_{k}$ given the measurements up to time $k, Z^{k}$, is called the posterior probability density function (pdf), denoted by $p\left(\boldsymbol{X}_{k} \mid \boldsymbol{Z}^{k}\right)$. An optimal Bayesian solution recursively maximizes the posterior pdf. In the case when (1) is linear and $\boldsymbol{w}$ and $\boldsymbol{n}$ are Gaussian white noise, a Kalman filter gives the optimal estimate.

\section{IMMERSIVE VR FOR BEHAVIOR EXPERIMENTS}

This section describes the generation of a moving stimulus on the computer screen as well as the setup for computing the three-dimensional pose and velocity of a viewer in realtime using a single camera.

\section{A. Looming stimulus}

We present a specific type of stimulus called a looming stimulus, which gives the appearance of an object approaching the viewer from a distance. Since we assume a perspective-projection model for the fish, the stimulus size increases as it gets closer. We produce the stimulus on a computer monitor placed to the side of a fish tank.

In addition to size, the looming stimulus will also change shape according to the perspective of the viewer. We use line of sight to define the perspective of the viewer. The line of sight may be different than the head orientation. We choose among the following ways to update the perspective of a viewer as a stimulus moves (Fig. 2): (1) set it at the center of the stimulus; (2) center it between the stimulus and the screen; and (3) center it on the screen. In human virtualreality applications, the perspective is often centered on the 

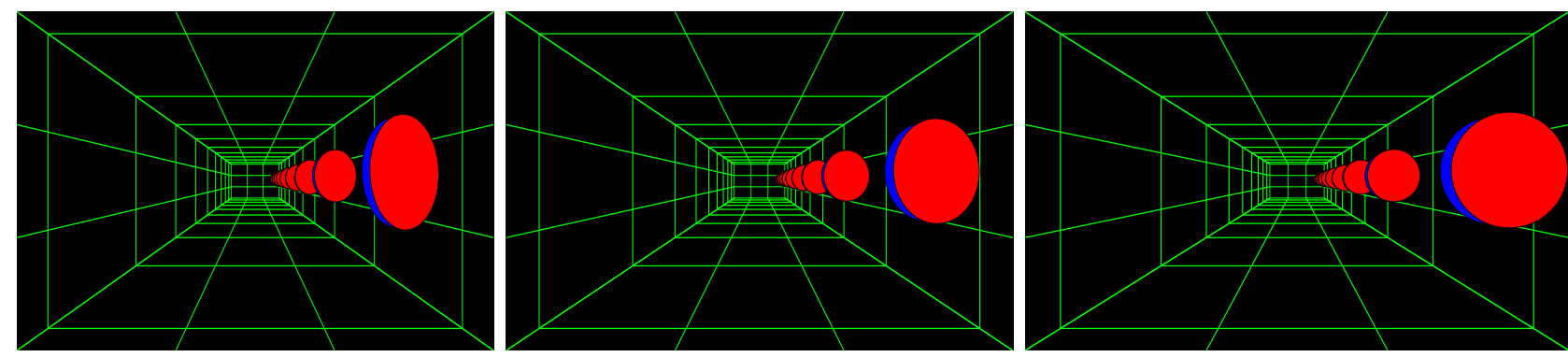

Fig. 2. A looming circular disc with different colored faces (red and blue) as it appears to the viewer: (left) looking at the disc, (middle) looking between the disc and the center of the screen, and (right) looking at the center of the screen.

screen because the viewer head pose is tracked [13]. For a looming stimulus, however, the perspective changes as the stimulus moves on the screen.

The azimuth and elevation of an approaching stimulus are based on the viewer's pose (see Fig. 1). The azimuth $-180^{\circ} \leq a \leq 180^{\circ}$, is the horizontal angle measured clockwise from the line between the viewer position and the projection of the viewer on the computer screen. The elevation $-90^{\circ} \leq h \leq 90^{\circ}$, is the angle between the stimulus and the horizontal plane. Due to the instantaneous viewer pose and screen size, the full range of azimuth and elevation may not be available to present the stimulus. Instead, with our real-time tracking system, the stimulus is conditionally presented based on the current viewer pose.

The size of the stimulus on the screen is computed as follows: given the real-world size $s_{d}$ of the stimulus, the distance $d_{v s}$ between the viewer and the stimulus center, and the distance $d_{v l}$ between the viewer and the screen along the line of sight, the screen dimension $s_{l}$ for the stimulus is

$$
s_{l}=\frac{s_{d}}{d_{v s}} d_{v l} .
$$

Equation (2) is similar to a perspective-projection model equation except that $d_{v l}$ and $d_{v s}$ vary as the stimulus moves. ( $d_{v l}$ does not change when the perspective is fixed or when the stimulus approaches the viewer along the line of sight.)

Taking the derivative of (2), we find the change in size of the stimulus on screen as a function of time:

$$
\begin{aligned}
\dot{s}_{l} & =-\dot{d}_{v s} \frac{s_{d}}{d_{v s}^{2}} d_{v l}+\frac{s_{d}}{d_{v s}} \dot{d}_{v l} \\
& =-\dot{d}_{v s} \frac{s_{l}}{d_{v s}}+\frac{s_{d}}{d_{v s}} \dot{d}_{v l} .
\end{aligned}
$$

Fig. 3 shows stimulus size as a function of time for constant acceleration and constant jerk (rate of change of acceleration). A related optical parameter $\theta$ called angular retinal image size [27], [7] may also be used to study the effects of the looming stimuli. The angular retinal image size is related to the size $s_{d}$ of the stimulus according to

$$
\theta=2 \tan ^{-1}\left(\frac{s_{d}}{2 d_{v s}}\right)=2 \tan ^{-1}\left(\frac{s_{l}}{2 d_{v l}}\right) .
$$

Variability in the appearance of a looming stimulus are introduced in terms of the starting and stopping position of the stimulus, its size, and dynamics.
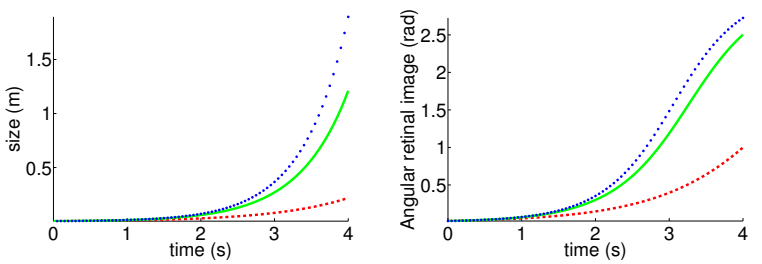

Fig. 3. (Left) Stimulus size for different types of motion: constant velocity (dashed red), constant acceleration (solid green), and constant jerk (blue dots). (Right) Corresponding change in angular retinal image size [7].

\section{B. Three-dimensional real-time tracking using a mirror}

We use the three-dimensional estimate of the fish pose to create an accurate representation of the stimulus at a given position on the screen. To obtain the three-dimensional estimate we need multiple views of the scene, so we mount a mirror on the side of the tank to obtain a second perspective. The view from the mirror is calibrated as a left-handed camera system. Since the overhead view and the reflection of the side of the tank is viewed from the same camera, we extract the three-dimensional estimate from a single frame. Each view within a frame is defined by a preset region of interest. To compute position, we project the head of the fish in the top view onto a line in the side view [28]. The head in the overhead view is marked as the center of the largest circle to fit within the top-view silhouette. (The centroid of the silhouette marks the body of the fish.) The pitch of the fish is found by the slope of the line that fits the side-view silhouette (in the mirror) in a least-squares sense. We use three constraints-two from projecting the estimate on the overhead view, and one from fitting the projected estimate to the line on the side view-to estimate the three-dimensional position of the head and body.

The estimate represents an input measurement to a Kalman filter with measurement noise values based on experimental data collected by tracking rigid known-sized objects. The filter state estimate $\boldsymbol{X} \in \mathbb{R}^{6}$ consists of the three-dimensional position and velocity of the head. Fish motion $\boldsymbol{F}$ is modeled as constant velocity with Gaussian disturbance, and $\boldsymbol{H}=$ $\left[\begin{array}{ll}\boldsymbol{I}_{3} & \mathbf{0}_{3}\end{array}\right]$, where $\boldsymbol{I}_{3}$ and $\mathbf{0}_{3}$ denote the $3 \times 3$ identity and zero matrices. Fish heading is computed instantaneously as the three-dimensional vector between the body and the head. We make two assumptions regarding fish movement and pose: (1) the fish head in our experiments is oriented in the direction of motion, and (2) the fish in our experiments turn 
and pitch but rarely roll. We validate the first assumption prior to presenting the stimulus by comparing the velocity vector with heading. Based on the second assumption we use the true vertical to compute the full fish pose [28].

A common problem associated with tracking within a glass tank is extraneous measurements arising due to reflections on the air-glass interface. Given a calibrated stereo camera pair, a measurement correspondence between the two views must satisfy the epipolar constraint [29]. We check the epipolar constraint for every combination of measurements within two views to filter out measurements caused by reflections.

\section{EXPERIMENTAL RESULTS}

\section{A. Materials and methods}

Giant danio 4-6 $\mathrm{cm}$ in body length were maintained in a 208 litre (55 gallon) tank, with approximately 45 fish in the tank at $30^{\circ} \mathrm{C}\left(83^{\circ} \mathrm{F}\right)$ on a 14 hours light / 10 hours dark photoperiod. Filtered and de-ionized water was provided by a pump system. Water quality was monitored regularly and was the same for experimental tanks.

The experimental setup consists of a $0.5 \mathrm{~m} \times 0.25 \mathrm{~m}$ $\times 0.30 \mathrm{~m}\left(20 " \times 10^{\prime} \times 12 "\right), 37.8$ litre (10 gallon) tank. The tank was backlit using diffused (1/4 stop) upwelling light from two 42 Watt lamps facing the floor and the wall opposite the mirror to create a uniform background. The fish did not exhibit aversive behavior to lighting conditions.

A 20 " screen for presenting the stimuli was set on one side of the tank at a resolution of $800 \times 600$ pixels and $60 \mathrm{~Hz}$ refresh rate. The video input to the screen was duplicated to a second 8 " screen that was installed facing up so that it could be used for verification. We used a Microsoft ${ }^{\mathrm{TM}}$ Cinema Lifecam at $640 \times 480$ pixel resolution mounted above a fish tank looking straight below. A mirror was mounted at an angle to the side of the tank as shown in Fig. 4. Intrinsic calibration was performed in air using a checkerboard and the MATLAB $^{\mathrm{TM}}$ calibration toolbox [30]. Extrinsic calibration was performed by immersing the checkerboard in water in view of the camera as well as the mirror. (The mirror was angled at $30^{\circ}$ to the tank surface.) The reprojection error for each camera view was in subpixels. Reconstruction error of $0.82 \pm 0.59 \mathrm{~mm}$ was determined empirically by triangulating 60 pairs of points ranging between 30 and $150 \mathrm{~mm}$ apart at various locations within the tank. This error, which is due to refraction from the air-water interface, is small because of the relatively small working volume [31]. Absolute position error of $1 \mathrm{~cm}$ was verified by projecting the position of a static object at various positions on the computer screen.

Threshold-based background subtraction from the first frame in each sequence was performed to isolate the fish in the region of interest for each camera view. The tracking algorithm (running at $15 \mathrm{~Hz}$ ) and looming stimulus (presented at $60 \mathrm{~Hz}$ ) were implemented in MATLAB using the Image Acquisition Toolbox. Fig. 5 shows images from a second camera used for independent verification of tracking and positioning of the stimulus with respect to the fish.

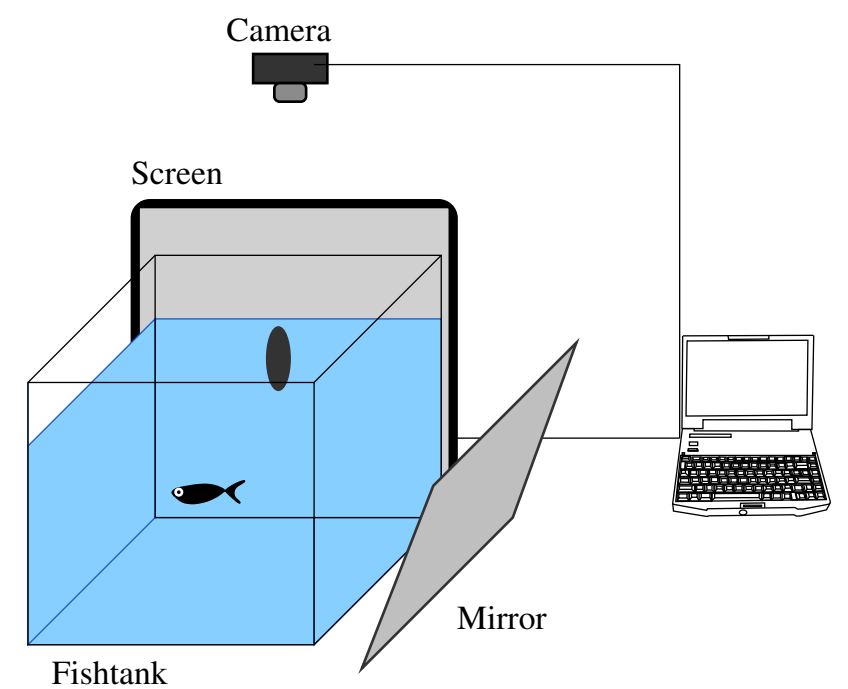

Fig. 4. Experimental setup

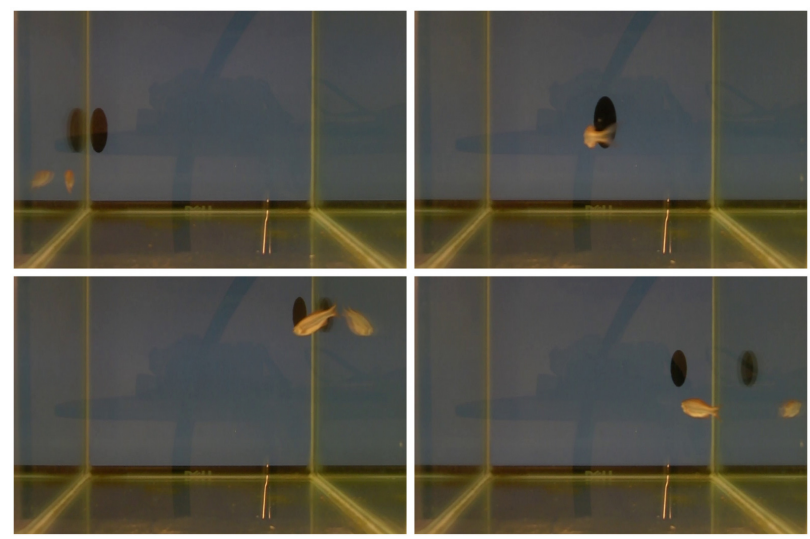

Fig. 5. View from a second camera for verification (see attached video).

\section{B. Behavioral experiments}

It is currently unknown what information fish extract from a looming stimulus. Evidence suggests that it may be a combination of optical parameters [7] one of which is the time-to-collision. Time to collision, $\tau$, is the time it would take the stimulus to reach the fish head. For small angles and a constant-velocity stimulus $\tau=\theta / \dot{\theta}$ [27]. Given an initial stimulus velocity and acceleration, we compute the value of $\tau$ based on the separation distance between the fish and the stimulus. We were unable to record eye movements, so the stimulus was presented based on fish pose.

We performed separate experiments to demonstrate the effectiveness and measure startle sensitivity. In the first experiment we presented the stimulus head-on to 4 different fish at random intervals and locations within the tank. This experiment served to validate the setup as well as to provide a method to detect startle responses automatically for the second experiment. In the second experiment 39 fish were presented an approaching stimulus from eight equally spaced regions around its head. The order of the regions and position within each region was sampled randomly. We describe below each of these experiments and the parameters used 
to perform the trials.

Experiment \#1: Effectiveness of VR setup We measured success in eliciting a startle response in freely roaming fish by presenting the stimulus head-on at random locations in the tank, and used fish acceleration to identify a startle response.

A stimulus was presented head-on every time the fish moved towards the screen $\left(-5^{\circ} \leq h \leq 5^{\circ}\right.$ and $-5^{\circ} \leq a \leq$ $5^{\circ}$ ). For each presentation of the stimulus, we determined whether a startle occurred and if so, the value of $\tau$ in the frame when the startle was detected. Trials consisted of presenting the stimulus to four different fish. Each session consisted of eight trials with a ten-minute habituation period and a randomized inter-trial interval of two to five minutes. The stimulus was started $50 \mathrm{~cm}$ away from the fish with an initial velocity of $100 \mathrm{~cm} / \mathrm{s}$ and a constant acceleration of $100 \mathrm{~cm} / \mathrm{s}^{2}$. The stimulus was an ellipsoid with axis lengths of $20 \mathrm{~cm}, 10 \mathrm{~cm}$ and $4 \mathrm{~cm} .{ }^{1}$

Experiment \#2: Investigation of startle response to VR stimuli The purpose of the second experiment was to measure the response kinematics and sensitivity of the giant danio as a function of orientation of a directed stimulus. The ultimate goal is to use this information to selectively startle a few fish in a school. We predicted that the fish has a lower probability of responding to a visual threat from behind. To test this prediction, the sphere around the fish head was divided into eight equal sections of $90^{\circ}$ azimuth and $90^{\circ}$ elevation. One section was randomly selected at the start of the trial and the fish tracked until the required azimuth and elevation range was reached. The stimulus was presented at a random location within that section. Trials consisted of a twenty-minute habituation period and randomized inter-trialintervals of three to nine minutes. Fish pose and velocity were validated at each timestep to ensure that (1) the fish heading and direction of motion was aligned (2) the fish was moving at a speed less than $150 \mathrm{~cm} / \mathrm{s}$ (3) the fish was more than one body length away from the tank walls. Stimulus properties were the same as in Experiment \#1.

\section{Results \& Discussion}

In Experiment \#1, the success rate of eliciting a startle response in fish for an oncoming stimulus was 94\% (30/32). Previous work in our laboratory with non virtual-reality stimuli resulted in about $30 \%-50 \%$ probability of response. Startle responses were verified visually. The fish acceleration during a startle ranged between $200-657 \mathrm{~cm} / \mathrm{s}^{2}$. (Values lying within this range in Experiment \#2 were marked as startle responses automatically.) The average time to collision for a head-on stimulus was $317 \pm 45 \mathrm{~ms}$.

In Experiment \#2, we investigated the startle response of a fish based on the directionality of a three-dimensional stimulus. A total of 267 stimulus presentations were recorded. The stimulus was presented uniformly from the left and right hemispheres of the fish (133 from the left and 134 from the right). For the top and bottom hemisphere, the

\footnotetext{
${ }^{1}$ All procedures were done according to protocol R 11-53 "Quantitative Analysis of Schooling Behavior" approved by the University of Maryland, College Park Institutional Animal Care and Use Committee.
}

presentations were not uniform (167 from the top and 100 from the bottom), due to tank size and the fact that the fish exhibited a preference for the bottom of the tank.

Fig. 6(a) shows the probability of a startle response calculated by taking the number of startle instances in a region divided by the total number of stimulus presentations in that region. Response probabilities varied between the eight regions, from $58 \%$ (slightly above chance levels) in the upper right front region to $94 \%$ in the back lower left region. When the data from separate regions was pooled, the probability of response to the front and to the right was $76 \%$ and the response probability to the back and the left was $80 \%$.

Fig. 6(b) shows the startle sensitivity map for each fish as a Mollweide (equal area) map projection. A total of 176 data points were selected out of 267 based on the threshold on acceleration. Although the mean ranks did not differ between the eight regions $(p=0.3209$, Kruskal-Wallis test), the fish appear to respond two body lengths sooner $(80 \mathrm{~ms})$ to a headon stimulus as compared to the ones coming from behind or above.
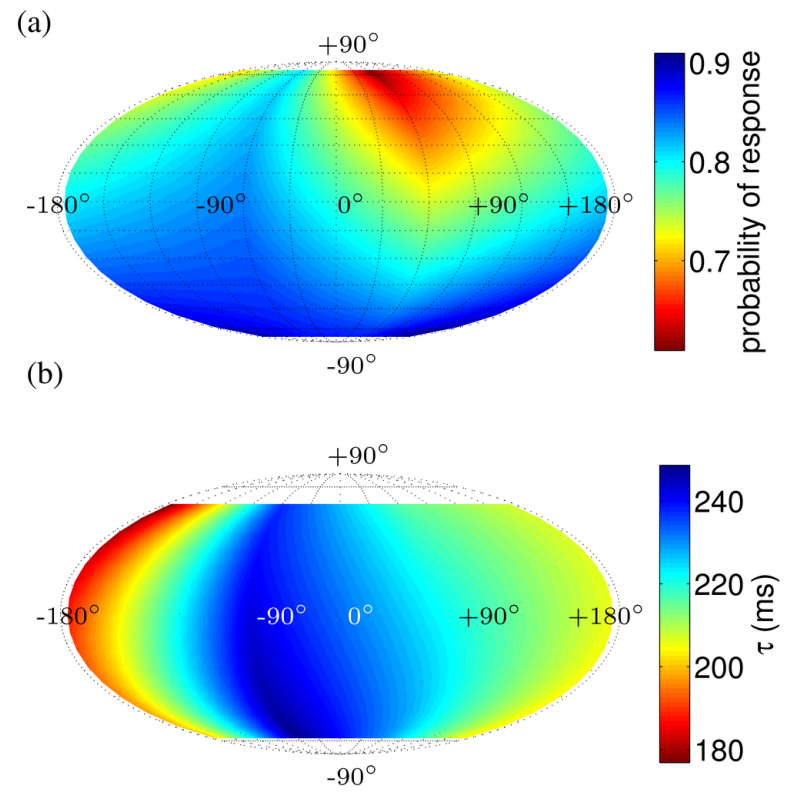

Fig. 6. (a) Probability of response and (b) mean value of time-to-collision $(\tau)$ interpolated between $90^{\circ}$ azimuth and $90^{\circ}$ elevation sections of the stimulus onset in the fish head frame for startle responses. The point $(0,0)$ marks the front of the fish, $(0,90)$ is above the fish head and $( \pm 180,0)$ is behind the fish.

We also recorded the trajectory of the individual fish away from the stimulus. Fig. 7 shows that greater number of responses move between $120^{\circ}-180^{\circ}$ away from a threat. Average change in depth was $30 \pm 26 \mathrm{~mm}$ from the fish start position.

\section{CONCLUSION}

We describe a novel virtual-reality framework to understand information transmission during an escape behavior 


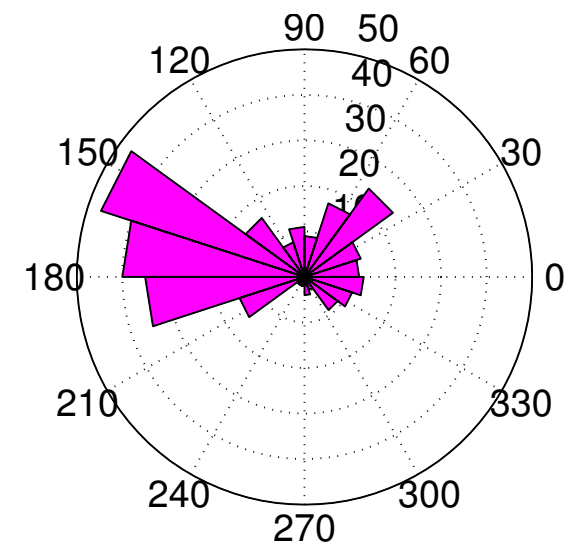

Fig. 7. Circular frequency distribution of escape trajectories of giant danio defined as the mean swimming direction of the fish twenty frames after a startle response with respect to stimulus orientation at rest. The stimulus is approaching from $0^{\circ}$ towards the center. Each concentric circle represents a frequency of 10 .

in fish, which is an important communication strategy in multi-agent robotics. We use the setup to investigate a fish's response to visual cues in a free-swimming environment. A looming stimulus, one that approaches the fish from a distance and grows in size, is used to validate the setup. The setup is fully automated and allows collection of high-volume datasets without any human intervention. Two experiments were performed to demonstrate its effectiveness. We demonstrate the success in eliciting a startle response by presenting the stimulus to 39 individual fish from different orientations. The startle sensitivity map allows us to tune the stimulus speed and size for targeting specific fish within a school. Such an approach, for example, would highlight the role of individual sensory modalities in a manipulative experiment where a specific number of fish are selectively targeted.

\section{ACKNOWLEDGMENTS}

The authors would like to thank Jennifer Lun for her work on the probability of startle response with a non virtual-reality stimulus. This material is based upon work supported by the National Science Foundation under Grant No. CMMI0954361.

\section{REFERENCES}

[1] F. Gerlotto, S. Bertrand, N. Bez, and M. Gutierrez, "Waves of agitation inside anchovy schools observed with multibeam sonar: a way to transmit information in response to predation," ICES J. of Marine Science, vol. 63, no. 8, pp. 1405-1417, 2006.

[2] R. Arkin, Behavior-based robotics. MIT Press, 1998

[3] A. Jadbabaie, J. Lin, and A. S. Morse, "Coordination of groups of mobile autonomous agents using nearest neighbor rules," in IEEE Trans. on Automatic Control, vol. 48, no. 6, 2003, pp. 988-1001.

[4] D. A. Paley, N. E. Leonard, R. Sepulchre, D. Grunbaum, and J. K. Parrish, "Oscillator models and collective motion," IEEE Control Systems Magazine, vol. 27, no. 4, pp. 89-105, 2007.

[5] H. Min and Z. Wang, "Design and analysis of Group Escape Behavior for distributed autonomous mobile robots," in Int. Conf. on Robotics and Automation. IEEE, 2011, pp. 6128-6135.

[6] R. Santer, P. Simmons, and F. Rind, "Gliding behaviour elicited by lateral looming stimuli in flying locusts," J.of Comparative Physiology $A$, vol. 191, no. 1, pp. 61-73, 2005.

[7] T. Preuss, P. E. Osei-Bonsu, S. A. Weiss, C. Wang, and D. S. Faber, "Neural representation of object approach in a decision-making motor circuit," J. of Neuroscience, vol. 26, no. 13, pp. 3454-3464, 2006.
[8] D. Oliva, V. Medan, and D. Tomsic, "Escape behavior and neuronal responses to looming stimuli in the crab Chasmagnathus granulatus (Decapoda: grapsidae)," J. of Exp. Biology, vol. 210, no. 5, 2007.

[9] J. Chen and H. Lee, "Application of virtual reality environment for studying rats cognitive behavior," Proc. of World Conf. on Educational Multimedia, pp. 4201-4207, 2007.

[10] A. D. Straw, K. Branson, T. R. Neumann, and M. H. Dickinson, "Multi-camera real-time three-dimensional tracking of multiple flying animals," J. Royal Society Interface, vol. 8, no. 56, pp. 395-409, 2010.

[11] H. Lee, R. Cherng, and C. Lin, "Development of a Virtual Reality Environment for Somatosensory and Perceptual Stimulation in the Balance Assessment of Children," Computers in Biology and Medicine, vol. 34, no. 8, pp. 719-733, 2004.

[12] A. Ward, "Quorum decision-making facilitates information transfer in fish shoals," Proc. of the Nat. Academy of Sciences, vol. 105, no. 19, pp. 6948-6953, 2008.

[13] J. Rekimoto, "A vision-based head tracker for fish tank virtual realityVR without head gear," in IEEE International Sym. on Virtual Reality, 1995, pp. 94-100.

[14] J. Mulder and R. Van Liere, "Enhancing fish tank VR," in Proc. of Virtual Reality, 2000, pp. 91-98.

[15] R. Eaton and D. Emberley, "How stimulus direction determines the trajectory of the Mauthner-initiated escape response in a teleost fish," Journal of experimental biology, vol. 161, no. 1, p. 469, 1991.

[16] H. Korn and D. Faber, "The Mauthner cell half a century later: a neurobiological model for decision-making?" Neuron, vol. 47, no. 1, pp. 13-28, 2005.

[17] G. Walls, The Vertebrate Eye and its Adaptive Radiation. Cranbrook Institute of Science, 1942.

[18] A. Sichert and J. van Hemmen, "How stimulus shape affects lateralline perception: Analytical approach to analyze natural stimuli characteristics," Biological Cybernetics, vol. 102, no. 3, pp. 177-180, 2010.

[19] R. Herikstad, J. Baker, J. Lachaux, C. Gray, and S. Yen, "Natural movies evoke spike trains with low spike time variability in cat primary visual cortex," J. of Neuroscience, vol. 31, no. 44, pp. 15844-15860, 2011.

[20] S. Hall, C. Wardle, and D. MacLennan, "Predator evasion in a fish school: test of a model for the fountain effect," Marine Biology, vol. 91, no. 1, pp. 143-148, 1986.

[21] V. Lepetit and P. Fua, "Monocular model-based 3D tracking of rigid objects: a survey," Foundations and Trends in Computer Graphics and Vision, vol. 1, no. 1, pp. 1-89, 2005.

[22] A. Yilmaz, O. Javed, and M. Shah, "Object tracking: A survey," ACM Computing Surveys (CSUR), vol. 38, no. 4, pp. 1-45, 2006.

[23] C. Wehrhahn, "Tracking and chasing in houseflies (Musca)," Biological Cybernetics, vol. 45, pp. 123-130, 1982.

[24] N. F. Hughes and L. H. Kelly, "New techniques for 3-D video tracking of fish swimming movements in still or flowing water," Canadian J. of Fisheries and Aquatic Sciences, vol. 53, no. 11, pp. 2473-2483, Nov. 1996.

[25] S. V. Viscido, J. K. Parrish, and D. Grünbaum, "Individual behavior and emergent properties of fish schools: A comparison of observation and theory," Marine Ecology Progress Series, vol. 10.3354/me, pp. 239-249, 2004.

[26] B. J. Laurel, C. J. Laurel, J. A. Brown, and R. S. Gregory, "A new technique to gather 3D spatial information using a single camera," $J$. of Fish Biology, vol. 66, pp. 429-441, 2005.

[27] N. Hatsopoulos, "Elementary computation of object approach by a wide-field visual neuron," Science, vol. 270, pp. 1000-1002, 1995.

[28] S. Butail and D. A. Paley, "Three-dimensional reconstruction of the fast-start swimming kinematics of densely schooling fish," J. Royal Soc. Interface, vol. 9, no. 66, pp. 77-88, 2011.

[29] R. Hartley and A. Zisserman, Multiple View Geometry in Computer Vision. Cambridge University Press, 2004.

[30] J.-Y. Bouguet. Camera Calibration Toolbox for Matlab. [Online]. Available: http://www.vision.caltech.edu/bouguetj/calib_doc/index.html

[31] V. Gourgoulis and N. Aggeloussis, "Reconstruction accuracy in underwater three-dimensional kinematic analysis," J. of Science and Medecine in Sport, vol. 11, no. 2, pp. 90-95, 2008. 